Maria Salinas*, Emilio Flores, Maite López-Garrigós, Maria Leiva-Salinas, Alberto Asencio, Javier Lugo and Carlos Leiva-Salinas

\title{
Computer-assisted interventions in the clinical laboratory process improve the diagnosis and treatment of severe vitamin B12 deficiency
}

https://doi.org/10.1515/cclm-2017-1116

Received November 30, 2017; accepted March 12, 2018; previously published online May 1, 2018

\begin{abstract}
Background: Severe vitamin B12 deficiency can result in serious complications if undiagnosed or untreated. Our aim was to test the efficacy of interventions in the laboratory process to improve the detection and the treatment of severe vitamin B12 deficiency.

Methods: Quasi-experimental investigation with a retrospective 7-year pre-intervention period and 29-month post-intervention follow-up in a university hospital. Two interventions were designed to improve the detection and treatment of subjects with vitamin B12 deficiency: the laboratory information system (LIS) automatically added seru vitamin B12 (s-vitamin B12) based on certain conditions; and created a comment in the report and scheduled an appointment with the general practitioner (GP). We calculated the number of new diagnoses of severe vitamin deficiency (s-vitamin B12 <73.8 pmol/L) and the proportion of identified patients that were correctly treated in the pre- and post-intervention periods. We compared the number of tests needed to detect a new case when ordered by GPs vs. added by the strategy. Finally, we investigated the economic cost of each new case.
\end{abstract}

\footnotetext{
*Corresponding author: Maria Salinas, PhD, Clinical Laboratory, Hospital Universitario de San Juan, Carretera Alicante-Valencia, s/n, 03550 San Juan de Alicante, Alicante, Spain; and Department of Biochemistry and Molecular Pathology, Universidad Miguel Hernandez, Elche, Spain, Phone: +34 965169463, Fax: +34 965169460, E-mail: salinas_mar@gva.es Emilio Flores: Clinical Laboratory, Hospital Universitario de San Juan, San Juan de Alicante, Alicante, Spain; and Department of Clinical Medicine, Universidad Miguel Hernandez, Elche, Spain Maite López-Garrigós and Javier Lugo: Clinical Laboratory, Hospital Universitario de San Juan, San Juan de Alicante, Alicante, Spain Maria Leiva-Salinas: Department of Biochemistry and Molecular Pathology, Universidad Miguel Hernandez, Elche, Spain Alberto Asencio: Primary Care Center of Muchamiel, Alicante-San Juan Health District, Muchamiel, Spain

Carlos Leiva-Salinas: Department of Radiology, University of Missouri, Columbia, MO, USA
}

Results: The strategy added 699 s-vitamin B12 and detected 66 new cases of severe vitamin deficiency. The number of tests needed to identify a new case when s-vitamin B12 was ordered by GPs was 187, as opposed to 10 when added through the intervention $(\mathrm{p}<0.001)$. The intervention reagent cost was $€ 26.7$ per new case. In the post-intervention cohort, $88 \%$ of patients were correctly treated, as opposed to $52 \%$ in the pre-intervention $(\mathrm{p}<0.001)$.

Conclusions: Interventions in the clinical laboratory process improved the diagnosis and treatment of severe vitamin B12 deficiency.

Keywords: clinical laboratory services; computer assisted diagnosis; primary health care; vitamin B12 deficiency.

\section{Introduction}

Since the introduction of the brain-to-brain loop concept for laboratory testing [1, 2], later known as total testing process [3], laboratory professionals should consider the steps that occur outside the laboratory in an effort to prevent errors related to the laboratory-clinician interface.

The terms "post-post" and "pre-pre” analytical steps were introduced to identify the activities associated with the clinician's initial choice of laboratory tests and the subsequent report interpretation and acting upon [4]. Both steps are more prone to errors than other pre-, post- [5-7] and even analytical activities [8]. In fact, inappropriateness of test request as well as the improper interpretation and utilization of results [3], represent a large percentage of total errors [9]. Although the request for laboratory tests has been extensively studied - and found to be highly variable [10], with both under- [11, 12] and over- [13] utilization - and several corrective strategies [12, 14-19] have been proposed, the post-post-analytical phase has been examined to a lesser degree. Furthermore, $25 \%-46 \%$ of laboratory errors are due to delayed or improper reaction to laboratory reporting, incorrect interpretation, inadequate follow-up plan or failure to order the appropriate consultation [20].

Vitamin B12 deficiency is associated with cognitive decline, dementia and Alzheimer's disease [21, 22], and 
may lead to DNA damage and altered methylation, both important risk factors for cancer. Older subjects are at the highest risk for vitamin B12 deficiency, as $10 \%-15 \%$ of people above 60 may present gastric atrophy and consequently hypocobalaminemia [23, 24]. Additionally, epidemiological studies found that $31 \%$ of depressed patients had serum vitamin B12 deficiencies [25] and one study found that patients with vitamin B12 deficiency are almost $70 \%$ more likely to suffer depression [26]. Additionally, commonly used treatments in primary care such as proton pump inhibitors and histamine-2 receptor antagonists, suppress the production of gastric acid and thus may lead to malabsorption of vitamin B12 [27].

In all, it is critical to identify and treat patients with vitamin B12 deficiency, given the major adverse effects and potential irreversible cognitive damage of this condition [28], the low economic cost and lack of toxicity of vitamin B12 treatment $[29,30]$ and, the technically easy and inexpensive way to detect it through measurement of serum vitamin B12 (s-vitamin B12).

We hypothesized that some meaningful low s-vitamin B12 results would unfortunately remain unacknowledged and not acted upon, and that corrective interventions would improve the proportion of patients that receive appropriate replacement therapy and the detected cases of severe vitamin B12 deficiency.

The purpose of this investigation is to test the efficacy of automatic interventions in the laboratory process to improve the detection and treatment of patients with severe vitamin B12 deficiency.

\section{Materials and methods}

\section{Study design}

A quasi-experimental investigation was conducted; a baseline preintervention cross-sectional study from January 1st 2008 to December 31st 2014, and a post-intervention follow-up from January 1st 2015 to May 31st 2017.

\section{Laboratory and hospital characteristics}

The clinical laboratory is located in a 370-bed suburban University Community Hospital that serves the population of the Health Department (HD) (234,551 inhabitants). It receives samples from inpatients, outpatients and primary care patients that are phlebotomized in nine different primary care centres (PCCs). Their samples are collected by couriers and delivered to the laboratory sample reception desk.

Primary care laboratory requests are made electronically from the patient's electronic medical record (EMR) by the general practitioners (GPs) and the reports are automatically sent from the laboratory information system (LIS) to the EMR. s-Vitamin B12 can only be requested in an individualized manner; the test does not belong to any laboratory profile.

\section{Participants}

We included all community inhabitants of the HD covered by the clinical laboratory. For analysis, we excluded those patients with vitamin B12 deficiency who passed away during the pre- and postintervention periods, and those that did not reside in our HD, as we did not have access to their EMR.

Ethical approval was not required for the study design.

\section{Intervention design}

In two meetings between the laboratory professionals and a GP coordinator representing the nine PCCs, we defined a new case with severe vitamin B12 deficiency when a patient had a new s-vitamin B12 $<73.8 \mathrm{pmol} / \mathrm{L}$ [31] regardless of whether s-vitamin B12 was or not previously measured in the past year. We identified the number of cases through a 7-year retrospective search in the LIS patient database. Based on our HD available resources and guidelines [32] a consensus was reached, and this new result was considered as (a) "communicated and received" when the result was made available in the EMR, and was checked by the GP; (b) "reviewed", when the GP treated the patient with any vitamin B12 supplements no later than 2 months after the phlebotomy and (c) "correctly interpreted and acted upon", when the patient received intramuscular (IM) treatment prescription [33] no later than 15 days after the phlebotomy. In situations when oral vitamin B12 treatment [34] was correctly prescribed instead of IM (e.g. vegetarians or IM treatment not accepted by the patient) these cases were considered "correctly interpreted and acted upon".

Two different interventions were designed to improve the detection (laboratory pre-pre-analytical-intervention) and treatment (postpost-analytical-intervention) of new cases with severe vitamin B12 deficiency.

Regarding the laboratory pre-pre-analytical intervention, the LIS automatically added s-vitamin B12 to the laboratory request of any primary care patient whose mean corpuscular volume (MCV) was higher than $100 \mathrm{fL}$ [35], when the former was not requested either in the current order or in the previous year.

Regarding the post-post-analytical-intervention, when a new case of severe vitamin B12 deficiency was identified, three actions were taken: the LIS automatically added a comment in the laboratory report ("Vitamin B12 therapy is recommended") and a patient appointment with the GP in the EMR. In addition to the electronic report in the EMR, a report was printed on garish color paper and shipped to the requesting GP to further alert him about the need of action.

\section{Outcome measures}

In both periods we computed the number of GP laboratory orders, total laboratory tests and s-vitamin B12 from primary care. We studied the number of new cases of severe vitamin B12 deficiency (s-vitamin B12 $<73.8 \mathrm{pmol} / \mathrm{L}$ ) and calculated the rate of severe vitamin B12 deficiency 
detection as the ratio of number of s-vitamin B12 measured per new detected cases. We compared the demographic data, s-vitamin B12, MCV and hemoglobin values in the subjects diagnosed with severe vitamin B12 deficiency.

Finally we calculated the number of s-vitamin B12 $<73.8 \mathrm{pmol} / \mathrm{L}$ results that were "communicated and received", "reviewed", and "correctly interpreted and acted upon".

In the post-intervention period, we studied how many subjects were identified, when vitamin B12 was ordered by GPs or added through the intervention, and calculated the number of tests needed to identify a new case, and compared the demographic data, s-vitamin B12, MCV and hemoglobin values in both groups.

We calculated the economic cost per new detected case through the intervention taking into account the reagent cost (€2.5 per test).

\section{Laboratory methods}

Three milliliters $(3 \mathrm{~mL})$ of blood sample were collected from each of the subjects into BD Vacutainer( $\left.{ }^{\circledR}\right) \mathrm{K}_{2}$ EDTA tubes (Becton, Dickinson and Company, Franklin Lakes, NJ, USA) to analyze CBC on Sysmex XE 2100 analyzer (Sysmex, Kobe, Japan). The concentration of s-vitamin B12 was measured from serum collected in BD Vacutainer $\left.{ }^{\circledR}\right)$ Serum Separating Tubes II Advance Tube (SST) (Becton, Dickinson and Company, Franklin Lakes, NJ, USA), using an immunoassay on Modular E170 (Roche Diagnostics, Indianapolis, IN, USA). The assay was subject to satisfactory internal and external quality control during the study. Between-batch coefficients of variation were $2.3 \%$ at 341 $\mathrm{pmol} / \mathrm{L}$, and $1.6 \%$ at $665 \mathrm{pmol} / \mathrm{L}$.

\section{Statistical analysis}

The sample size was calculated from the prevalence from prior studies, taking into account a 3\% frequency of vitamin B12 deficiency [20]. The $95 \%$ confidence level indicated a minimum cohort size of 146 patients with vitamin B12 deficiency for both the pre- and postintervention periods, considering our study population $(234,551$ inhabitants), assuming a $20 \%$ loss.

The statistical analyses were performed with SPSS (SPSS Inc., Chicago, IL, USA). The statistical analysis included a descriptive analysis of the variables: the distribution, the mean, standard deviation, median and percentile 25-75. The comparative study by two percentages was done by the $\chi^{2}$-test. A two-sided $\mathrm{p} \leq 0.05$ rule was utilized as the criterion for rejecting the no difference.

\section{Results}

\section{Patient population}

During the 7-year pre-intervention period, the number of total laboratory tests from primary care increased by $30 \%$, while the demand for s-vitamin B12 tripled along this period (6647 in 2014, and 2083 in 2008) (Table 1). Patients

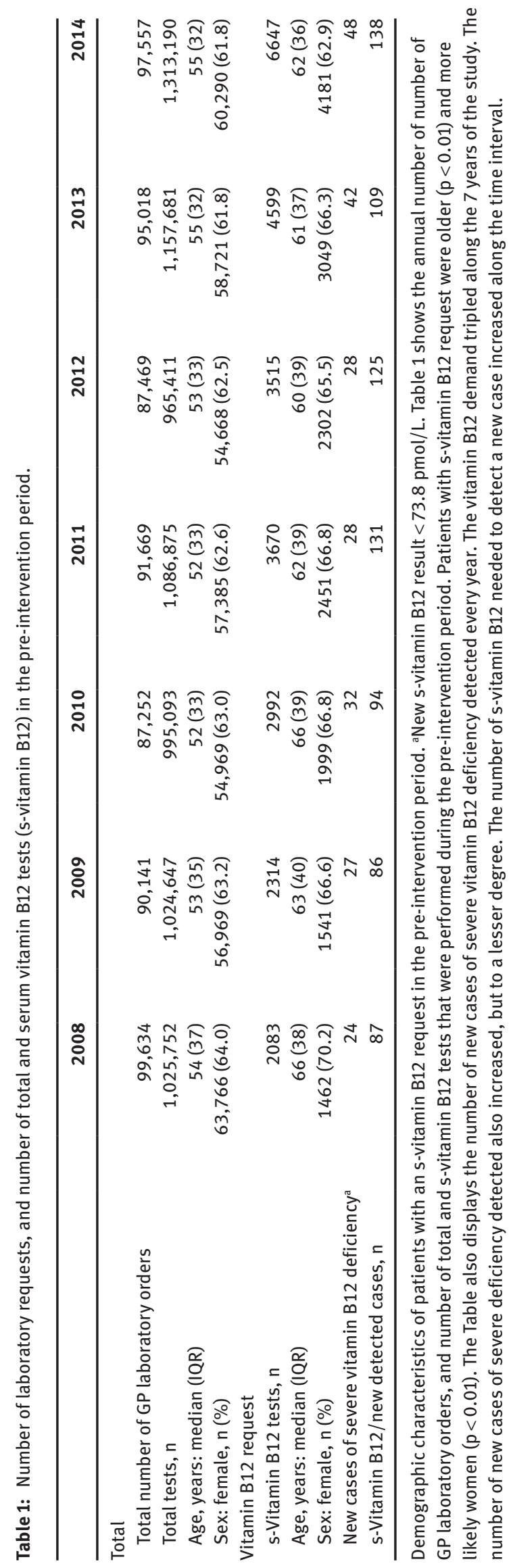


with s-vitamin B12 request were older $(\mathrm{p}<0.001)$ and predominantly women $(\mathrm{p}<0.001)$. The number of new cases of severe vitamin B12 deficiency doubled over that interval (48 in 2014, and 24 in 2008); accordingly, the number of s-vitamin B12 needed to identify a new case (rate of detection of severe vitamin B12 deficiency) increased to 138 in 2014 from 87 in 2008 (Table 1).

In the post-intervention period, 226,906 laboratory requests containing 5,071,530 total laboratory tests were received from primary care.

In the pre-intervention period, 229 new cases of severe vitamin B12 deficiency were identified; 31 died and one did not live in our HD, resulting in a pre-intervention cohort of 197 patients. Among those, 85 (43.1\%) had anemia, and 118 (59.9\%) had macrocytosis (Table 2). Among patients with anemia $4.1 \%$ had microcytic and $36.0 \%$ had normocytic anemia.

In the post-intervention period, 192 new cases of severe vitamin B12 deficiency were detected. Out of those, two died and nine did not live in our HD, resulting in a post-intervention cohort of 181 . Among those, 67 (37\%) had anemia, and $102(56.1 \%)$ had macrocytosis (Table 2). Among patients with anemia 3.3\% had microcytic and $40.6 \%$ had normocytic anemia. There were no significant differences in demographic characteristics and laboratory tests results between "new cases of severe vitamin B12 deficiency" in the pre- and postintervention groups.

Table 2: Demographic characteristics and analytical pattern of new cases of vitamin B12 deficiency in the pre- and post-intervention cohorts.

\begin{tabular}{|c|c|c|}
\hline & \multicolumn{2}{|r|}{ Total } \\
\hline & $\begin{array}{r}\text { Pre-intervention } \\
n=197\end{array}$ & $\begin{array}{r}\text { Post-intervention } \\
n=181\end{array}$ \\
\hline $\begin{array}{l}\text { Age, years: median } \\
\text { (P25-P75) }\end{array}$ & $77(66-84)$ & $76(65-84)$ \\
\hline Older > 65 years, $\mathrm{n}(\%)$ & $144(73.1)$ & $136(75.1)$ \\
\hline $\begin{array}{l}\text { s-Vitamin B12, pmol/L: } \\
\text { median (P25-P75) }\end{array}$ & $49.1(34.2-61.0)$ & $50.8(36.2-64.1)$ \\
\hline MCV, fL: mean (SD) & $102.8(14.2)$ & $99.0(11.2)$ \\
\hline Macrocytosis, n (\%) & $118(59.9)$ & $102(56.1)$ \\
\hline $\begin{array}{l}\text { Hemoglobin, g/dL: } \\
\text { median (P25-P75) }\end{array}$ & $12.3(11.0-1.6)$ & $12.8(11.5-14.2)$ \\
\hline Anemia, n (\%) & $85(43.1)$ & $67(37.1)$ \\
\hline $\begin{array}{l}\text { Female }<12 \mathrm{~g} / \mathrm{dL} \\
\text { Male }<13 \mathrm{~g} / \mathrm{dL}\end{array}$ & & \\
\hline
\end{tabular}

Table 2 compares the demographic and analytical characteristics of patients in the in pre-intervention and in post-intervention cohorts. There were no statistically significant differences.

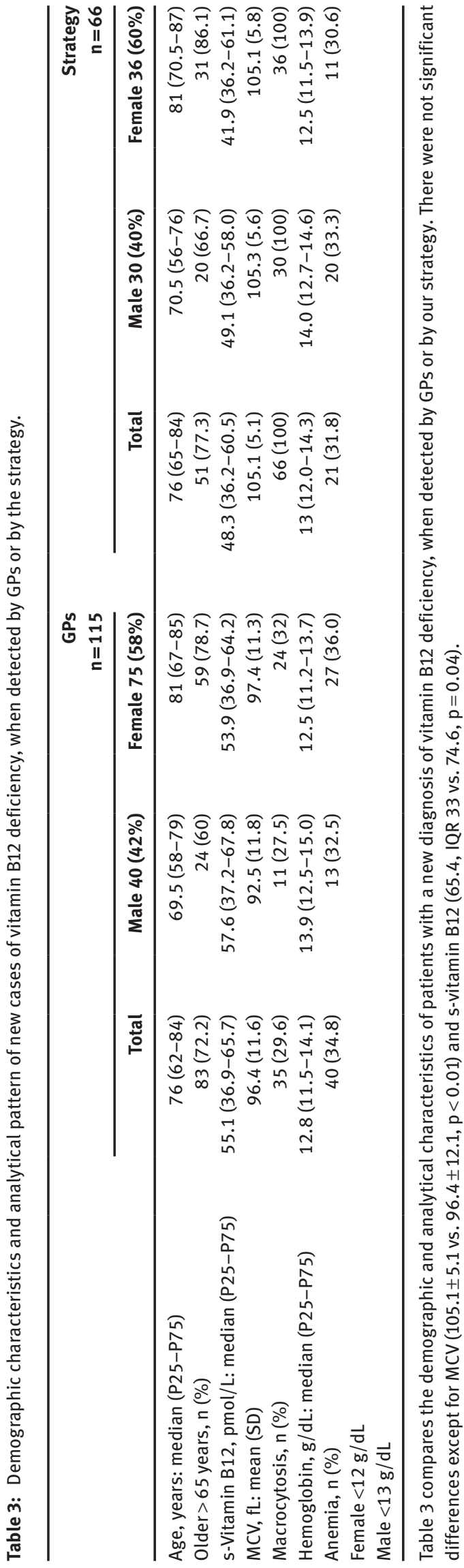




\section{Impact of the laboratory pre-pre-analytical intervention}

In the post-intervention period, 22,352 s-vitamin B12 were requested by the GPs, and 699 were added by our strategy. Among the 181 new cases of severe vitamin B12 deficiency, s-vitamin B12 was requested by the GPs in 115 and added by the LIS according to the intervention in the remaining 66 . The number of tests needed to identify a new case when s-vitamin B12 was ordered by GPs was 194, as opposed to 10 when added through the intervention $(p<0.001)$. The demographic characteristics and laboratory results in patients detected by the intervention were not significantly different from the ones detected by GPs, except for MCV (105.1 \pm 5.1 vs. $96.4 \pm 12.1, \mathrm{p}<0.001)$ and s-vitamin B12 (65.4, IQR 33 vs. 74.6, IQR 39, $p=0.04$ ); these results are shown in Table 3.

Regarding the economic perspective, the total reagent cost to measure the additional 699 s-vitamin B12 was 1750.1€; which resulted in a cost of €26.7 per new patient detected.

\section{Impact of the post-post-analytical intervention}

In the pre-intervention period, all 197 (100\%) low s-vitamin B12 results were communicated and received, 164 (82\%) were reviewed, and 103 (52\%) were correctly interpreted and acted upon (Figure 1).
In the post-intervention cohort, all 181 low s-vitamin B12 results were communicated and received as well, 177 (98\%) were reviewed, and 159 (88\%) were correctly "interpreted and acted upon", as opposed to $52 \%$ in the pre-intervention cohort $(\mathrm{p}<0.001)$.

\section{Discussion}

Strategies impacting the laboratory pre-pre- and postpost-analytical phases efficiently identified subjects with severe vitamin B12 deficiency that would have otherwise probably remained undiagnosed, and resulted in a more effective clinical management.

Through the automatic register of s-vitamin B12 in the agreed conditions, a new case of severe vitamin B12 deficiency was detected for every 10 measured tests.

Throughout the pre-intervention period, requests for s-vitamin B12 tripled, with more cases of deficiency detected. However, with time, more s-vitamin B12 requests were needed to detect a new case of severe vitamin B12 deficiency; i.e. more cases were detected but in a less efficient way. The cause of such an increase is unknown but it could reflect increased use of s-vitamin B12 for monitoring replacement. This could potentially be improved with a pre-pre-analytical intervention such as the use of GP education or clinical decision support software.

In the laboratory pre-pre-analytical intervention, making use of the LIS and patient data bases, an

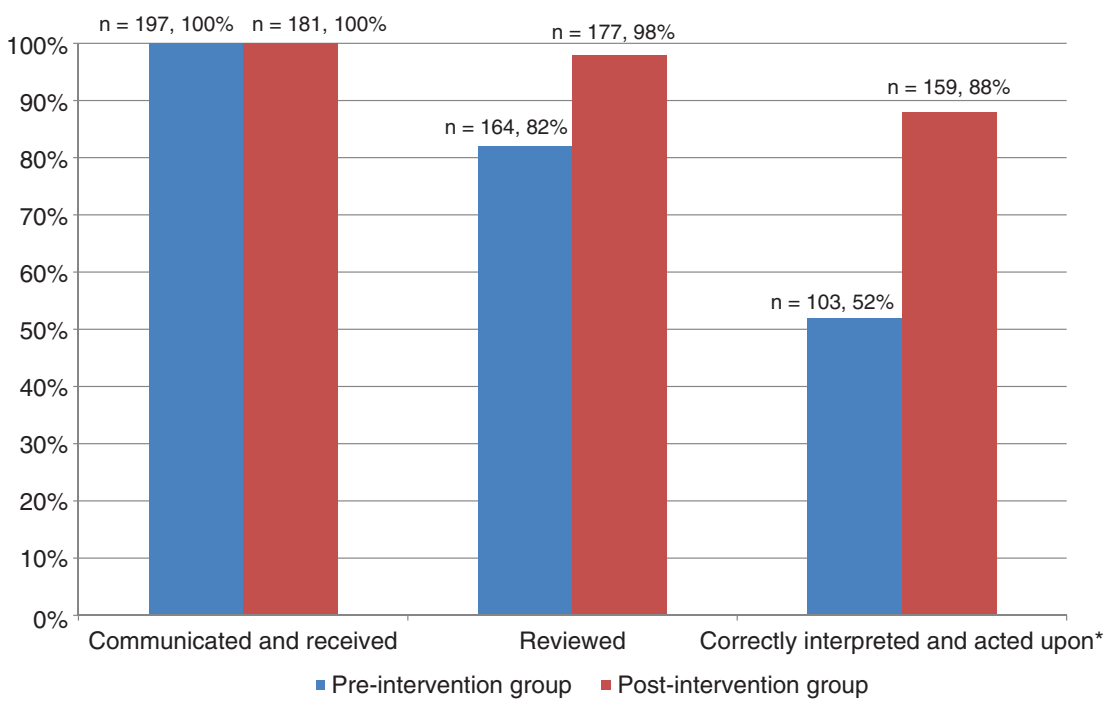

Figure 1: Management of new cases of severe vitamin B12 deficiency by General Practitioners (GPs). ${ }^{*} \mathrm{p} \leq 0.01$. Communicated and received: $\mathrm{s}$-Vitamin B12 result was made available in the electronic medical record, and was checked by the GP. Reviewed: Patient was treated with any vitamin B12 supplement no later than 2 months after phlebotomy. Correctly interpreted and acted upon: the patient received intramuscular treatment prescription no later than 15 days after phlebotomy. 
automatic intervention was designed and implemented in order to register s-vitamin B12 in primary care patients' requests when $\mathrm{MCV}>100 \mathrm{fL}$. We chose the number of new cases of severe vitamin B12 deficiency and cost per case as outcome indicators [16]. Sixty-six new cases were detected at a cost of $€ 26.7$ per case. Given the potential devastating consequences of vitamin B12 deficiency, the above price, and the lack of adverse effects of replacement therapy, this cost per case detected seems very reasonable and affordable for any health system, and suggests that the intervention was successful.

Regarding the post-post-analytical intervention, the addition of the interpretative comments in the laboratory report, reporting information in addition to simple data [36, 37], dramatically improved patient management; most s-vitamin B12 results of new cases of severe vitamin B12 deficiency were correctly interpreted and acted upon.

Despite the fact that all results were communicated and received, around half of the patients were not adequately treated in the pre-intervention period. This probably reflects that most clinicians were unaware of the low s-vitamin B12 results or their important consequences. This has been reported in the past, as a high percentage of physicians did not respond to elevated calcium results by writing a note or ordering another test [38].

The study had certain limitations. First, we were probably only impacting a proportion of patients with vitamin B12 deficiency given the fact that just under half $(40 \%-$ 44\%) of patients in both cohorts did not present with a low MCV, and some patients with B12 deficiency may have been missed with serum B12 above the $73.8 \mathrm{pmol} / \mathrm{L}$ cut off. Moreover a weakness of the strategy could be the neglect of other factors that impact MCV, such as iron deficiency and liver dysfunction. Second, we could not study how many patients had resolution of macrocytosis, or anemia or symptoms improvement. Third, as the standardization of s-vitamin B12 assays is not completed [39] and there is inconsistency in the literature regarding s-vitamin B12 cut-offs for the diagnosis of vitamin B12 deficiency in the general population [40] methyl-malonic acid, total homocysteine or holotranscobalamin could have been measured to document deficiency, although s-vitamin B12 levels below 73.8 pmol/L are usually associated with clinical deficiency [41]. Our population was largely elderly and the strategy may not apply to younger adults. Given time frames for "reviewed" and "correctly interpreted and acted upon" are relatively short, it is conceivable that the response would have been greater if more time was allowed for patient follow-up; this is another potential limitation of the study. Finally, the calculated economic costs of the study may not apply to other settings, as the authors' laboratory belongs to a Public Health Network, where reagent prices are relatively low.

Despite the fact that B12 deficiency can be treated with vitamin replacement, in many cases results indicating B12 deficiency were not acted upon correctly, thus potentially influencing patient outcome and societal wellbeing. Accordingly, it is crucial not only to make sure that the laboratory result has been communicated, received and reviewed by the right provider, but also that it has been interpreted correctly and the subsequent advisable clinical action(s) are indeed completed [42, 43].

Strategies impacting the laboratory total testing process efficiently detected occult new cases of severe vitamin B12 deficiency and improved treatment of such patients.

Author contributions: All the authors have accepted responsibility for the entire content of this submitted manuscript and approved submission.

Research funding: None declared.

Employment or leadership: None declared.

Honorarium: None declared.

Competing interests: The funding organization(s) played no role in the study design; in the collection, analysis, and interpretation of data; in the writing of the report; or in the decision to submit the report for publication.

\section{References}

1. Lundberg GD. Acting on significant laboratory results. J Am Med Assoc 1981;245:1762-3.

2. Lundberg GD. How clinicians should use the diagnostic laboratory in a changing medical world. Clin Chim Acta 1999;280:3-11.

3. Plebani M, Lippi G. Closing the brain-to-brain loop in laboratory testing. Clin Chem Lab Med 2011;49:1131-3.

4. Laposata M, Dighe A. "Pre-pre” and "post-post" analytical error: high-incidence patient safety hazards involving the clinical laboratory. Clin Chem Lab Med 2007;45:712-9.

5. Gandhi TK, Kachalia A, Thomas EJ, Puopolo AL, Yoon C, Brennan TA, et al. Missed and delayed diagnoses in the ambulatory setting: a study of closed malpractice claims. Ann Intern Med 2006;145:488-96.

6. Hickner J, Graham DG, Elder NC, Brandt E, Emsermann CB, Dovey $S$, et al. Testing process errors and their harms and consequences reported from family medicine practices: a study of the American Academy of Family Physicians National Research Network. Qual Saf Health Care 2008;17:194-200.

7. Kachalia A, Gandhi TK, Puopolo AL, Yoon C, Thomas EJ, Griffey R, et al. Missed and delayed diagnoses in the emergency department: a study of closed malpractice claims from 4 liability insurers. Ann Emerg Med 2007;49:196-205.

8. Wahls TL, Cram PM. The frequency of missed test results and associated treatment delays in a highly computerized health system. BMC Fam Pract 2007;8:32. 
9. Plebani M. Exploring the iceberg of errors in laboratory medicine. Clin Chim Acta 2009;404:16-23.

10. van Walraven C, Naylor CD. Do we know what inappropriate laboratory utilization is? A systematic review of laboratory clinical audits. J Am Med Assoc 1998;280:550-8.

11. Salinas M, López-Garrigós M, Uris J, Leiva-Salinas C. A study of the differences in the request of glycated hemoglobin in primary care in Spain: a global, significant, and potentially dangerous under-request. Clin Biochem 2014;47:1104-7.

12. Salinas M, López-Garrigós M, Pomares F, Lugo J, Asencio A, López-Penabad L, et al. Serum calcium (S-Ca), the forgotten test: preliminary results of an appropriateness strategy to detect primary hyperparathyroidism (pHPT). Bone 2013;56:73-6.

13. Salinas M, López-Garrigós M, Flores E, Uris J, Leiva-Salinas C. Potential over request in anemia laboratory tests in primary care in Spain. Hematology 2015;20:368-73.

14. Salinas M, López-Garrigós M, Flores E, Leiva-Salinas M, Lugo J, Pomares FJ, et al. Automatic laboratory-based strategy to improve the diagnosis of type 2 diabetes in primary care. Biochem Med 2016;26:121-8.

15. Salinas M, López-Garrigós M, Asencio A, Batlle E, Minguez $M$, Lugo J, et al. Strategy to improve the request of uric acid in primary care: preliminary results and evaluation through process and outcome appropriateness indicators. Clin Biochem 2014;47:467-70.

16. Salinas M, López-Garrigós M, Flores E, Leiva-Salinas M, Asencio A, Lugo J, et al. Managing inappropriate requests of laboratory tests: from detection to monitoring. Am J Manag Care 2016;22:e311-6.

17. Feldman LS, Shihab HM, Thiemann D, Yeh H-C, Ardolino M, Mandell S, et al. Impact of providing fee data on laboratory test ordering: a controlled clinical trial. JAMA Intern Med 2013;173:903-8.

18. Wong ET, Lincoln TL. Ready! Fire! . . Aim! An inquiry into laboratory test ordering. J Am Med Assoc 1983;250:2510-3.

19. Berwick DM, Coltin KL. Feedback reduces test use in a health maintenance organization. J Am Med Assoc 1986;255:1450-4.

20. Hawkins R. Managing the pre- and post-analytical phases of the total testing process. Ann Lab Med 2012;32:5-16.

21. Moore E, Mander A, Ames D, Carne R, Sanders K, Watters D. Cognitive impairment and vitamin B12: a review. Int Psychogeriatrics 2012;24:541-56.

22. Chen H, Liu S, Ji L, Wu T, Ma F, Ji Y, et al. Associations between Alzheimer's disease and blood homocysteine, vitamin B12, and folate: a case-control study. Curr Alzheimer Res 2015;12: 88-94.

23. Baik HW, Russell RM. Vitamin B12 deficiency in the elderly. Annu Rev Nutr 1999;19:357-77.

24. Allen LH. How common is vitamin B-12 deficiency? Am J Clin Nutr 2009;89:693S-6.

25. Carney MW, Sheffield BF. Serum folic acid and B12 in 272 psychiatric in-patients. Psychol Med 1978;8:139-44.
26. Tiemeier H, van Tuijl HR, Hofman A, Meijer J, Kiliaan AJ, Breteler MM. Vitamin B 12, Folate, and Homocysteine in depression: the Rotterdam Study. Am J Psychiatry 2002;159:2099-101.

27. Lam JR, Schneider JL, Zhao W, Corley DA. Proton pump inhibitor and histamine 2 receptor antagonist use and vitamin B12 deficiency. J Am Med Assoc 2013;310:2435-42.

28. Lachner C, Steinle NI, Regenold WT. The neuropsychiatry of vitamin B 12 deficiency in elderly patients. J Neuropsychiatry Clin Neurosci 2012;24:5-15.

29. Carmel R, Chanarin I, Carmel R, Carmel R, Healton E, Savage $D$, et al. How I treat cobalamin (vitamin B12) deficiency. Blood 2008;112:2214-21.

30. Berlin H, Berlin R, Brante G. Oral treatment of pernicious anemia with high doses of vitamin B 12 without intrinsic factor. Acta Med Scand 1968;184:247-58.

31. Snow CF. Laboratory diagnosis of vitamin B12 and folate deficiency: a guide for the primary care physician. Arch Intern Med 1999;159:1289-98.

32. Ruiz de Adana R. Anemia perniciosa. La Coruña: Fisterra.com. Available from: http://www.fisterra.com/guias-clinicas/anemiaperniciosa/.

33. Langan RC, Goodbred AJ. Vitamin B12 deficiency: recognition and management. Am Fam Physician 2017;96:384-9.

34. Bolaman Z, Kadikoylu G, Yukselen V, Yavasoglu I, Barutca S, Senturk T. Oral versus intramuscular cobalamin treatment in megaloblastic anemia: a single-center, prospective, randomized, open-label study. Clin Ther 2003;25:3124-34.

35. Ruiz de Adana R, Marco MA. Macrocitosis. La Coruña: Fisterra. com. Available from: https://www.fisterra.com/guias-clinicas/ macrocitosis/.

36. Salinas M, López-Garrigós M, Uris J. Towards laboratory knowledge, not data, in $70 \%$ of clinical decision-making. What "knowledge management" can add to clinical practice? Clin Chem Lab Med 2011;49:1389-90.

37. Salinas M, López-Garrigós M, Asencio A, Lugo J, Gutiérrez M, Flors L, et al. Alert value reporting: a new strategy for patient safety. Clin Biochem 2013;46:245-9.

38. Howanitz PJ, Cembrowski GS. Postanalytical quality improvement: a College of American Pathologists Q-Probes study of elevated calcium results in 525 institutions. Arch Pathol Lab Med 2000;124:504-10.

39. İspir E, Serdar MA, Ozgurtas T, Gulbahar O, Akın KO, Yesildal F, et al. Comparison of four automated serum vitamin B12 assays. Clin Chem Lab Med 2015;53:1205-13.

40. Aparicio-Ugarriza R, Palacios G, Alder M, González-Gross M. A review of the cut-off points for the diagnosis of vitamin B12 deficiency in the general population. Clin Chem Lab Med 2015;53:1149-59.

41. Stabler SP. Vitamin B12 deficiency. N Engl J Med 2013;368:149-60.

42. Walz SE, Darcy TP. Patient safety \& post-analytical error. Clin Lab Med 2013;33:183-94.

43. Skendzel LP. How physicians use laboratory tests. J Am Med Assoc 1978;239:1077-80. 